\title{
Intrinsic Pinning in Layered Antiferromagnetic Superconductor
}

\author{
Tomasz Krzysztoń \\ Institute for Low Temperatures and Structure Research, Polish \\ Academy of Sciences,50-950 Wrocław,Poland ${ }^{1}$
}

${ }^{1}$ E-mail krzyszto@apollo.int.pan.wroc.pl 


\begin{abstract}
The intrinsic pinning in high-temperature superconductor with long range antiferromagnetic order of rare-earth ions confined to the isolating planes is described. The interaction of antiferromagnetic and superconducting subsystems in the mixed state may lead to the creation of spin-flop domain along the vortex core. It is shown that the behavior of several physical quantities such as magnetization, activation energy, current-voltage characteristic and flux creep changes when the direction of the external field changes in the basal a-b plane. It is also shown that the decay of the trapped flux is logarithmic function of time.
\end{abstract}




\section{INTRODUCTION}

The problem of interplay between long-range magnetism and superconductivity was studied thoroughly several years ago in Chevrel phases, rhodium borides and rhodium stannides [1]. On the contrary, this phenomenon has not attracted much attention in the high-temperature superconductors (HTS). Both competitive phenomena coexist in classical superconductors because $4 \mathrm{f}$ electrons of rare-earth (RE) atoms responsible for magnetism and 4d electrons of molybdenum chalcogenide clusters (rhodium boride or rhodium stannide clusters) responsible for superconductivity are spatially separated from each other. The situation seems to be very similar in layered HTS. Here magnetic order is produced by the regular lattice of RE ions occupying isolating layers electrically isolated from the superconducting $\mathrm{Cu}-\mathrm{O}$ planes. Therefore spin interaction between the local magnetic moments and the conduction electrons is to weak to inhibit superconductivity. The typical example of that layered system is $\mathrm{ErBa}_{2} \mathrm{Cu}_{3} \mathrm{O}_{7}$. This compound has tetragonal unit cell with small orthorhombic distortion in the a-b plane [2]. The Er ions form two sublattice antiferromagnetic structure of magnetic moments lying parallel and antiparallel to the b direction in the a-b plane [3]. Recently discovered RE nickel boride-carbides [⿴囗十] may serve as an another example of layered magnetic superconductors. The structure of these compounds is similar to that of HTS and consists of RE-C layers separated by $\mathrm{Ni}_{2} \mathrm{~B}_{2}$ sheets. For example in $\mathrm{ErNi}_{2} \mathrm{~B}_{2} \mathrm{C}$ [0] the antiferromagnetic structure is associated with magnetic moments of $\mathrm{Er}^{+3}$ ions, which order below $6 \mathrm{~K}$ in a transversely polarized planar sinusoidal structure propagating along a or b axis with Er moments parallel to the a or b axis respectively.

In this paper we consider the structure shown on Fig.1 that we believe simulates real structure of many antiferromagnetic layered superconductors. It consists of superconducting layers of thickness $\mathrm{d}_{s}$ and magnetic moments of $\mathrm{RE}$ ions running parallel and antiparallel to the $\mathrm{b}$ axis in the isolating layers of thickness $d_{i}, d=d_{s}+d_{i} \approx d_{i}$.

An antiferromagnet with two sublattices shows different magnetization behavior in an external magnetic field applied parallel or perpendicular to the easy axis 6]. The main difference is that antiferromagnetic configuration $(\mathrm{AF})$ is unstable in perpendicular field. When the external field is applied parallel to the magnetic moments the AF phase is stable up to the critical field $H_{T}$ above which spin-flop phase develops. This was the basic idea put forward to explain strange behavior of $\mathrm{DyMo}_{6} \mathrm{~S}_{8}$ in applied magnetic field [7]. Neutron scattering experiments [8] have shown that this compound develops long range AF state in the presence of superconductivity. Unexpectedly some ferromagnetic peaks were observed in the mixed state when the applied 
field exceeded 200 Oe, considerably below $H c_{2}$. The idea of induced spinflop transition near the core of the vortex was introduced to explain these observations. The ferromagnetic-like order confined along the vortices was belived to be responsible for additional peaks above 200 Oe.

In the present model (see also [9]) we come across two following situations:

1. The external magnetic field is pointing in the $\mathrm{b}$ direction and exceeds $H_{T}$ in the vortex core. Then the magnetic subsystem in the core undergoes the spin-flop transition.

2. The external magnetic field is applied along the a direction. Then the AF alignment is unstable against spin-flop transformation and the vortex state is created in the uniform spin-flop phase.

Assume the first case. The field intensity in the vortex core in the low vortex density regime doubles the value of the external field intensity [10]. The vortices are created in the uniform antiferromagnetic medium if the condition $H c_{1}<H<\frac{1}{2} H_{T}$ is fulfilled. When the external field reaches $\frac{1}{2} H_{T}$, the magnetic field inside the core is $H_{T}$ whereas outside it decreases from this value. In this way the spin-flop domain appears along the vortex. Finally, depending on the external field intensity, there can be two types of vortices in the specimen. On the other hand, in the case 2 , the vortices do not change their structure and are similar to that in nonmagnetic HTS.

The magnetic field aligned parallel to the conducting planes makes the vortex lattice to accommodate itself to the layer structure so that the vortex cores lie in between the superconducting sheets. A current density $j$ flowing along the planes exerts a Lorentz force on the vortices in the c direction. Intrinsic pinning barriers are formed on strongly superconducting layers. The vortices loose large amount of condensation energy when they move across the barriers. Depending on the external field intensity and its direction in the $a-b$ plane we deal with vortices of two types. It is interesting therefore to see how changes the intrinsic pinning barrier during the creation of magnetic domain along the vortex.

\section{LONDON EQUATIONS}

The electrodynamics of layered superconductors is based on the LawrenceDoniach model that in the London approximation gives the following functional of the free energy of superconducting subsystem [11]

$$
F_{s}=\frac{\varphi_{0}^{2} d}{8 \pi^{2} \mu_{0} \lambda_{a b}^{2}} \sum_{n} \int\left\{\left(\nabla \Phi_{n}+\frac{2 \pi}{\varphi_{0}} \mathbf{A}_{p}\right)^{2}+\frac{2}{r_{j}^{2}}\left[1-\cos \left(\chi_{n}\right)\right]\right\} d^{2} r
$$


where $r_{j}=d\left(\lambda_{c} / \lambda_{a b}\right), \mathbf{A}=\left(\mathbf{A}_{p}, A_{z}\right), \mathbf{A}_{p}=\left(A_{x}, A_{y}\right), \varphi_{0}$ denotes the flux quantum, $\mu_{0}$ the magnetic permeability of the vacuum, $\lambda_{c}, \xi_{c}$ and $\lambda_{a b}, \xi_{a b}$ the magnetic field penetration depth and the coherence length parallel and perpendicular to the layers respectively. The parameter $r_{j}$ plays the role of the effective radius of the vortex core. The term with cosine gives the Josephson current due to the gauge invariant phase difference between the layers

$$
\chi_{n}=\Phi_{n+1}-\Phi_{n}+\frac{2 \pi}{\varphi_{0}} \int_{(n+1) d}^{n d} A_{z} d z .
$$

The antiferromagnetic two sublattice subsystem with single ion anisotropy is described with the following free energy functional:

$F_{m}=d_{i} \sum_{n} \int\left\{J \mathbf{M}_{1 n} \mathbf{M}_{2 n}-K \sum_{i=1}^{2} M_{i n \perp}-\gamma \sum_{i=1}^{2} \sum_{\alpha=x, y, z}\left(\nabla M_{i n \alpha}\right)^{2}\right\} f_{n, n+1}(z) d^{2} r$,

where $\mathbf{M}_{n}=\mathbf{M}_{1 n}+\mathbf{M}_{2 n}$ is the sum of the magnetization vectors of the sublattices in the $\mathrm{n}$-th insulating layer, $M_{i n \perp}$ is the component along the anisotropy axis of the magnetization sublattice vector in the n-th layer, $J$ denotes the exchange constant between two sublattices, $K$ is the single ion anisotropy constant, $\sqrt{\gamma}$ is the magnetic stiffness length, the factor $f_{n, n+1}(z)=1$ for insulating layers and 0 otherwise, and $M=\left|\mathbf{M}_{1 n}\right|=\left|\mathbf{M}_{2 n}\right|$. Finally we add the magnetic field energy to obtain the free energy of the entire system

$$
F=F_{s}+F_{m}+\frac{\mu_{0}}{2} \int \mathbf{H}^{2} d^{2} r d z .
$$

The coupling between the magnetic and superconducting subsystem is supposed to be electromagnetic. This means that both order parameters are coupled through the vector potential A

$$
\begin{gathered}
\mathbf{B}=\operatorname{rot} \mathbf{A}=\mu_{0} \mathbf{H}+\mathbf{M}, \\
|\Psi|^{2} \approx j_{s}=|\operatorname{rot} \mathbf{H}|,
\end{gathered}
$$

where $\mathbf{B}$ ( in MKSA units) is the vector of a magnetic flux density ( magnetic induction) and $\mathbf{H}$ is the vector of a thermodynamic magnetic field intensity. The analysis of a stability of the functional (3) against the spin-flop transition gives the thermodynamical critical field of the upper limit of the $\mathrm{AF}$ configuration equal to

$$
H_{T}=M_{0} \sqrt{K(J-K)}
$$


The London equations resulting from (4) are following:

$$
\begin{aligned}
& B_{x}+\lambda_{c}^{2} \frac{\partial}{\partial y} \operatorname{rot}_{z}(\mathbf{B}-\mathbf{M})-\lambda_{a b}^{2} \frac{\partial}{\partial z} \operatorname{rot}_{y}(\mathbf{B}-\mathbf{M})=\varphi_{0} \delta(y) \delta(z), \\
& B_{y}+\lambda_{a b}^{2} \frac{\partial}{\partial z} \operatorname{rot}_{x}(\mathbf{B}-\mathbf{M})-\lambda_{c}^{2} \frac{\partial}{\partial x} \operatorname{rot}_{z}(\mathbf{B}-\mathbf{M})=0 \\
& B_{z}+\lambda_{a b}^{2} \frac{\partial}{\partial x} \operatorname{rot}_{y}(\mathbf{B}-\mathbf{M})-\lambda_{a b}^{2} \frac{\partial}{\partial y} \operatorname{rot}_{x}(\mathbf{B}-\mathbf{M})=0 .
\end{aligned}
$$

This equations should be supplemented by the appropriate set of differential equations describing the spatial distribution of magnetization. Simple conjecture, as shall be seen later, can make the calculations less complex and at the same time does not oversimplify the problem. The London model of continuous superconductors may be used at length scales larger than the coherence length, i.e. the core dimension. The structure of a vortex lying in the a-b plane in a layered superconductor with Josephson coupling between adjacent layers resembles the Abrikosov's one except that the order parameter does not vanish anywhere [12]. Instead there exists a region, $r_{j}$ along the plane and $d$ perpendicular to it, where the Josephson current $j_{z}$ is of the order of the critical current. In this region, which plays the role of the vortex core, the London model fails. Away from the core the streamlines of the shielding supercurrents, which also represents contours of constant magnetic field, are elliptical except for the zigzags due to the intervening insulating layers(Fig.2). It is not crucial what kind of transition is inherent in the magnetic subsystem: spin-flop, metamagnetic or paramagnetic. The main fact is the appearance of a magnetic domain with an induced magnetic moment $M$ along the vortex. We make the above mentioned conjecture by assuming [9] that the magnetic moment is constant across the domain

$$
|\mathbf{M}|=\left\{\begin{array}{cll}
M & \text { if } & \rho<\rho_{m} \\
0 & \text { if } & \rho>\rho_{m}
\end{array},\right.
$$

where $\rho_{m}$ is the dimensionless radius of the magnetic domain in the coordinate system of the elliptical cylinder $\left(x=x, y=\lambda_{c} \rho \cos \varphi, z=\lambda_{a b} \rho \sin \varphi\right)$.

Then, the set of equations (7) have the following solutions [9]

$$
\begin{aligned}
B_{P} & =C_{1} I_{0}(\rho)+C_{2} K_{0}(\rho) \text { for } \rho<\rho_{m} \\
B_{A F} & =\frac{\mu_{0} H_{T}}{K_{0}\left(\rho_{m}\right)} K_{0}(\rho) \text { for } \rho>\rho_{m}
\end{aligned}
$$

where $B_{P}$ and $B_{A F}$ denote the magnetic induction inside and outside the 
domain respectively. The constants $C_{1}$ and $C_{2}$ are calculated using (8) and the flux quantization condition to obtain

$$
\begin{aligned}
C_{1} & =\frac{B_{T} \rho_{m} I_{1}\left(\rho_{m}\right)-D\left(\rho_{m}\right) I_{0}\left(\rho_{m}\right)}{1-I_{0}\left(\rho_{m}\right)} \\
C_{2} & =\frac{D\left(\rho_{m}\right) K_{0}\left(\rho_{m}\right)+B_{T}\left[\rho_{m} K_{1}\left(\rho_{m}\right)-1\right]}{1-I_{0}\left(\rho_{m}\right)} \\
D\left(\rho_{m}\right) & =\frac{\varphi_{0}}{2 \pi \lambda_{a b} \lambda_{c}}-\mu_{0} H_{T} \frac{\rho_{m} K_{1}\left(\rho_{m}\right)}{K_{0}\left(\rho_{m}\right)}
\end{aligned}
$$

where $K_{0}, K_{1}, I_{0}, I_{1}$ denote the modified Bessel functions whereas $B_{T}=$ $M+\mu_{0} H_{T}$. Now we can think of $M$ as of experimental parameter. To calculate $\rho_{m}$ one must first derive the line tension of the vortex and then look for its minimum with respect to the domain radius. Standard calculations give

$$
\rho_{m}^{2} \approx \frac{5 \varphi_{0}}{8 \pi \lambda_{a b} \lambda_{c} B_{T}}
$$

It is easy to see that almost all flux quantum is captured in the magnetic domain.

\section{ACTIVATION ENERGY}

The first quantitative approach toward intrinsic pinning in layered superconductors was based on the observation that the superconducting order parameter should have a periodic spatial variation across the layers [13]. For the present considerations, however, the method of critical nucleus developed in 14] is much more convenient. The activated nucleus consists of a kinkantikink excitation, that is, a vortex line segment is thrown to the adjacent layer, thereby creating two pancake vortices of opposite "vorticity", as shown on Fig.3. The activation energy can be regarded as the energy barrier for intrinsic pinning. Depending on the magnitude of the driving current density the process may continue as the single vortex activation or the activation of the vortex bundle. First, consider the activation of a segment of a vortex to the neighboring interlayer spacing (Fig.3). The energy associated with this process can be written as:

$$
U=\delta E+V_{K,-K}(R)-\left(j-j_{0}\right) \varphi_{0} d R
$$

The subscript "a" or "b" (of $U, \delta E$ and $j_{0}$ ) indicates that this quantity is calculated for a or b direction in the plane. $\delta E$ is the amount of condensation 
and magnetic domain energy that is lost at two points of the layer threaded by the kinks separated by a distance $\mathrm{R}$ (see Fig.3). $V_{K,-K}(R)$ is the kinkantikink interaction energy. The term proportional to the driving current $j$ is due to the Lorentz force. The term proportional to $j_{0}$ is the energy associated with the distortion of the line due to the formation of nucleus. This term can be estimated from the simple considerations 14

$$
j_{0} \varphi_{0} d \sim \frac{1}{2} \int d y d z C(y, z)\left(\frac{\partial u_{z}}{\partial z}\right)^{2}
$$

where the Fourier transform of the compression modulus is given by [15]

$$
C\left(k_{y}, k_{z}\right)=\frac{B^{2}}{\mu_{0}\left(1+\lambda_{a b}^{2} k_{z}^{2}+\lambda_{c}^{2} k_{y}^{2}\right)} .
$$

By taking $d y d z \sim \varphi_{0} / B, u_{z} \sim d, \frac{\partial}{\partial z} \sim k_{z} \sim k_{y}\left(\lambda_{c} / \lambda_{a b}\right)$ the integral can be estimated as follows

$$
j_{0 a}=\frac{B d}{4 \lambda_{a b}^{2}} .
$$

In the $\mathrm{b}$ direction, however, we have an additional contribution from the magnetic domain $d y d z \sim 5 \varphi_{0} / 8 B_{T}$ according to (11), so we get

$$
j_{0 b}=j_{0 a}+\frac{5 d B_{T}}{128 \lambda_{a b}^{2}} .
$$

As the current density $j$ drops below $j_{0}$ a single-vortex line can no longer be activated due to the confinement energy provided by the vortex lattice. The energy $\delta E$ is calculated from (7) with the right-hand sides representing the vortex cores:

$$
\begin{aligned}
& \left\{|x|>\frac{R}{2}, y=0, z=0\right\},\left\{|x|<\frac{R}{2}, y=0, z=-d\right\} \\
& \text { and }\left\{x= \pm \frac{R}{2}, y=0,0<z<-d\right\} .
\end{aligned}
$$

The solution is then substituted to the free energy functional (4). Taking the limit $R \rightarrow \infty$ we exclude the energy of the kink-antikink interaction. The calculations are involved, so we write down only the results.

$$
\delta E_{a}=2 d \epsilon_{0} \ln \frac{r_{j}}{\xi_{a b}}
$$




$$
\delta E_{b}=d \epsilon_{b} \ln \frac{r_{j}}{\xi_{a b}},
$$

where $\epsilon_{0}=\varphi_{0}^{2} /\left(16 \pi^{2} \mu_{0} \lambda_{a b}^{2}\right), \epsilon_{b}=\frac{77}{64} \epsilon_{0} \ln \left[\varphi_{0} /\left(\pi r_{j}^{2} B_{T}\right)\right]$.

The energy of kink-antikink interaction was calculated in [14]

$$
V_{K,-K}(R)=-\frac{d^{2} \epsilon_{0}}{2 \lambda_{a b}} f\left(\frac{R}{\lambda_{c}}\right)
$$

where $f\left(\frac{R}{\lambda_{c}}\right)=\left\{\begin{array}{ccc}\left(\lambda_{c} / R\right)-\ln \left(r_{j} / \xi_{a b}\right) & \text { for } & r_{j}<<R<<\quad \lambda_{c} \\ 2\left(\lambda_{c} / R\right)^{3} \exp \left(-R / \lambda_{c}\right) & \text { for } & R>>\lambda_{c}\end{array}\right.$.

We introduce the Ginzburg-Landau critical current density $j_{G L}=4 \epsilon_{0} /\left(\varphi_{0} \xi_{a b} 3 \sqrt{3}\right)$ and the quantity $I_{a, b}=2\left(j-j_{0 a, b}\right) /\left(j_{G L} 3 \sqrt{3}\right)$, then (12) can be rewritten in the following way

$$
\begin{aligned}
U_{a} & =2 d \epsilon_{0}\left\{\ln \frac{r_{j}}{\xi_{a b}}+I_{a} \frac{R}{\xi_{a b}}-\frac{d}{4 \lambda_{a b}} f\left(\frac{R}{\lambda_{c}}\right)\right\}, \\
U_{b} & =d\left\{\epsilon_{b} \ln \frac{r_{j}}{\xi_{a b}}+2 \epsilon_{0} I_{b} \frac{R}{\xi_{a b}}-\frac{d \epsilon_{0}}{2 \lambda_{a b}} f\left(\frac{R}{\lambda_{c}}\right)\right\} .
\end{aligned}
$$

The critical size of the nucleus $R_{c}$ is given as a minimum of (18) with respect to $R$. In the approximation $r_{j}<<R<<\lambda_{c}$ corresponding to the current regime $\xi_{c} d / \lambda_{a b}^{2}<<I_{a, b}<<\xi_{c} / d$ we get

$$
\begin{aligned}
R_{c a, b}^{2} & =\xi_{a b}^{2} \frac{d}{4 I_{a, b} \xi_{c}}, \\
U_{a}^{c} & =2 d \epsilon_{0}\left\{\ln \left(\frac{r_{j}}{\xi_{a b}}\right)-\sqrt{\frac{d I_{a}}{\xi_{c}}}\right\}, \\
U_{b}^{c} & =d\left\{\epsilon_{b} \ln \left(\frac{r_{j}}{\xi_{a b}}\right)-2 \epsilon_{0} \sqrt{\frac{d I_{b}}{\xi_{c}}}\right\} .
\end{aligned}
$$

For the opposite case $R>>\lambda_{c}$ and $\xi_{c} d / \lambda_{a b}^{2}>>I_{a, b}$

$$
\begin{aligned}
R_{c a, b} & =\lambda_{c} \ln \left(\frac{d \xi_{c}}{I_{a} \lambda_{a b}^{2}}\right), \\
U_{a}^{c} & =2 d \epsilon_{0}\left\{\ln \left(\frac{r_{j}}{\xi_{a b}}\right)-\frac{\lambda_{a b} I_{a}}{\xi_{c}} \ln \left(\frac{d \xi_{c}}{I_{a} \lambda_{a b}^{2}}\right)\right\}, \\
U_{b}^{c} & =d\left\{\epsilon_{b} \ln \left(\frac{r_{j}}{\xi_{a b}}\right)-\frac{\lambda_{a b} I_{b}}{\xi_{c}} \ln \left(\frac{d \xi_{c}}{I_{b} \lambda_{a b}^{2}}\right)\right\} .
\end{aligned}
$$


When the driving current drops below $j_{0}$ the critical nucleus is $3 \mathrm{D}$ object (Fig.4). In our case it is a parallelepiped of the height $R$ along the bundle and of the section $S$ across it. The activation energy is a sum of the volume energy due to the Lorentz force and the surface energy.

$$
U_{a, b}=-j B d R S+\delta E_{a, b}\left(\frac{B S}{\varphi_{0}}\right)+j_{0 a, b} d R \sqrt{B S \varphi_{0}} .
$$

The second term is the loss of condensation energy (and magnetic domain energy in the case of $\mathrm{b}$ direction) on both surfaces perpendicular to the bundle multiplied by the number of vortices threading these surfaces. The third term is the elastic energy released in the surface parallel to the shifted vortex $j_{0 a, b} d R \varphi_{0}$ multiplied by the number of shifted vortices $\sqrt{B S / \varphi_{0}}$ (one vortex per plane). The critical nucleus is then $S_{c}=\left(\varphi_{0} / B\right)\left(j_{0 a, b} / j\right)^{2}$, $R_{c}=\delta E_{a, b} /\left(j d \varphi_{0}\right)$, and the activation energy is

$$
U_{a, b}^{c}=\delta E_{a, b}\left(\frac{j_{0 a, b}}{j}\right)^{2}
$$

\section{MOTION OF THE FLUX}

The resistive mechanism in the mixed state is determined by the activation process leading to magnetic flux motion (creep). This motion induces electric field which can be observed on the current-voltage characteristic. We consider the motion of activated kinks along the layers of the length $L$ along the magnetic field direction. Assume that each one can reach the boundary of the sample before the new one is created. The mean electric field associated with this motion is given by

$$
E=B P L d S_{c},
$$

where $P$ is the activation probability per unit volume and unit time. For thermal activation this probability is given by $P=\alpha \exp \left(-U_{c} / k_{B} T\right)$. There is however a crossover temperature $T_{0}$ [16] below which quantum tunneling of vortices is dominating. The probability for quantum tunneling is given by $P=\beta \exp \left(-U_{c} / k_{B} T_{0}\right)$ and remains finite even for $T=0$. The temperature $T_{0}$ depends on the driving current, critical current, upper critical field, and resistivity in the normal state. For our purposes it is worth to note that the quantity $\exp \left(-U_{c} / k_{B} T_{0}\right)$ will contribute only for driving currents of the order of the critical current [16]. The Neel temperature for layered antiferromagnetic superconductors varies from hundreds of $\mathrm{mK}(0.6 \mathrm{~K}$ for $\mathrm{ErBa}_{2} \mathrm{Cu}_{3} \mathrm{O}_{7}$ ) to several Kelvin $\left(6.8 \mathrm{~K}\right.$ for $\mathrm{ErNi}_{2} \mathrm{~B}_{2} \mathrm{C}$ ) and therefore both 
mechanisms of activation are present in these compounds. The preexponential factors and $T_{0}$ cannot be calculated in the framework of thermodynamic considerations alone. Fortunately, it was shown in [17] that the activation probability of macroscopic quantum excitations is proportional to $j^{3}$. Thus we can assume that $P=\alpha_{0} j^{3} \exp \left(-U_{c} / k_{B} T\right)$. Now we can calculate the current-voltage characteristics for the current density regimes considered previously. For $j<<j_{0}$

$$
\begin{aligned}
& E_{a}=\varphi_{0} d L \alpha_{0} j_{0 a}^{2} j \exp \left\{-\frac{\delta E_{a}}{k_{B} T}\left(\frac{j_{0 a}}{j}\right)^{2}\right\}, \\
& E_{b}=\varphi_{0} d L \alpha_{0} j_{0 b}^{2} j \exp \left\{-\frac{\delta E_{b}}{k_{B} T}\left(\frac{j_{0 b}}{j}\right)^{2}\right\} .
\end{aligned}
$$

This almost linear dependence of $E$ on $j$ indicates that the resistive mechanism of bundle activation follows Ohm law.

For $j>>j_{0}$ and $\xi_{c} d / \lambda_{a b}^{2}<<I_{a, b}<<\xi_{c} / d$

$$
\begin{aligned}
& E_{a}=\varphi_{0} d L \alpha_{0} j^{3} \exp \left\{-\frac{\delta E_{a}}{k_{B} T}+\frac{2 d \epsilon_{0}}{k_{B} T} \sqrt{\frac{d I_{a}}{\xi_{c}}}\right\} \\
& E_{b}=\varphi_{0} d L \alpha_{0} j^{3} \exp \left\{-\frac{\delta E_{b}}{k_{B} T}+\frac{2 d \epsilon_{0}}{k_{B} T} \sqrt{\frac{d I_{b}}{\xi_{c}}}\right\} .
\end{aligned}
$$

For $j>>j_{0}$ and $\xi_{c} d / \lambda_{a b}^{2}>>I_{a, b}$

$$
\begin{aligned}
& E_{a}=\varphi_{0} d L \alpha_{0} j^{3} \exp \left\{-\frac{\delta E_{a}}{k_{B} T}+\frac{2 d \epsilon_{0}}{k_{B} T} \frac{\lambda_{a b} I_{a}}{\xi_{c}} \ln \left(\frac{d \xi_{c}}{\lambda_{a b}^{2} I_{a}}\right)\right\}, \\
& E_{b}=\varphi_{0} d L \alpha_{0} j^{3} \exp \left\{-\frac{\delta E_{b}}{k_{B} T}+\frac{2 d \epsilon_{0}}{k_{B} T} \frac{\lambda_{a b} I_{b}}{\xi_{c}} \ln \left(\frac{d \xi_{c}}{\lambda_{a b}^{2} I_{b}}\right)\right\} .
\end{aligned}
$$

We can also calculate the rate of flux creep due to the thermal activation of vortices. To do this consider hollow cylindrical sample of a radius $r$ and the wall thickness $l<<r$ placed in the magnetic field $B_{e x}>B_{c 1}$ applied parallel to the cylinder axis. The sample has the trapped field $B_{i n}$ inside the hole and trapped flux $\Phi=\left(B_{i n}-B_{e x}\right) \pi r^{2}$. According to the Faraday's law electric field due to the change of the trapped flux is equal to $\left(\mu_{0} / 2\right) \operatorname{lr}(d j / d t)$. Combining this result with (23) we have finally

$$
B P L d S_{c}+\frac{1}{2} \mu_{0} l r \frac{d j}{d t}=0 .
$$


This equation can be solved analytically only in the case of the weak currents. Consequently for excitations in the form of bundle of vortices (27) is written as

$$
\Omega j \exp \left\{-\frac{\delta E_{a, b}}{k_{b} T}\left(\frac{j_{0 a, b}}{j}\right)^{2}\right\}+\frac{d j}{d t}=0,
$$

where $\Omega=\varphi_{0} \alpha_{0} j_{0}^{2} /\left(\mu_{0} \gamma\right)$ and $\gamma=r l /(L d)$ is the factor determined by the geometry of the sample. The solution of (28) is given in terms of exponential integrals and for the case of $j_{0 a, b} / j-1<<1$ it can be approximated as:

$$
\frac{j(0)}{j(t)}-1=\frac{\Phi(0)}{\Phi(t)}-1=\frac{k_{B} T}{2 \delta E_{a, b}}\left(\frac{j(0)}{j_{0 a, b}}\right)^{2} \ln \left(1+\omega_{a, b} t\right),
$$

where

$$
\omega_{a, b}=\frac{4 \varphi_{0} \alpha_{0} \delta E_{a, b}}{\mu_{0} \gamma}\left(\frac{j_{0 a, b}}{j(0)}\right) \exp \left\{-\frac{\delta E_{a, b}}{k_{b} T}\left(\frac{j_{0 a, b}}{j(0)}\right)^{2}\right\} .
$$

This result is in agreement with the experiments on nonmagnetic HTSC ( see [18]). For $0<<t<<1 / \omega$ the change of trapped flux is linear in time and for $t>>1 / \omega$ logarithmic. In antiferromagnetic superconductors, however, we see additional change of characteristic frequency as the magnetic field changes its direction in the a-b plane.

\section{CONCLUSIONS}

The current $j_{0}$ plays very important role in all above calculations. For the following values $d \sim 10^{-9} \mathrm{~m}, \xi_{c} \sim 3 \times 10^{-10} \mathrm{~m}, \xi_{a b} \sim 3 \times 10^{-9} \mathrm{~m}, r_{j} \sim 10^{-8} \mathrm{~m}$ and their typical temperature dependence we can estimate

$$
\frac{j_{0 a}}{j_{G L}} \sim \frac{B d \xi_{a b}}{\varphi_{0}} \sim 10^{-3} B \sqrt{\frac{T_{c}}{T_{c}-T}} .
$$

Since the depairing current is of the order of $10^{13}\left[T_{c} /\left(T_{c}-T\right)\right]^{3 / 2}$ then the current $j_{0 a}$ is of the order of $10^{10} B\left[T_{c} /\left(T_{c}-T\right)\right]$. Although there are no precise measurements of spin-flop transition in the antiferromagnetic high temperature superconductors, we assume that $\mu_{0} H_{T} \sim 40 \mathrm{mT}$. The typical value of $5.5 \mu_{B}$ per rare-earth atom per unit cell gives $M \sim 0.37 T$. It is possible now to estimate the change of $j_{0}$ due to the creation of spin-flop domain along the vortex : 


$$
\frac{j_{0 a}}{j_{0 b}} \sim 1+0.625 \frac{B_{T}}{B} \sim 3
$$

providing that $H_{T} / H_{c 1}-1<<1$. Similar considerations show that the characteristic frequency of flux creep changes of order of magnitude when the spin-flop domain is created.

\section{ACKNOWLEDGEMENTS}

This work was financially supported by Komitet Badan Naukowych under grant 2PO3B17109. 


\section{References}

[1] Ø. Fischer and M. B. Maple, eds. Superconductivity in ternary compounds (Springer, Berlin, 1983).

[2] J. W. Lynn, J. Alloys and Compounds 181 (1992) 419.

[3] J. W.Lynn, T. W. Clinton, W-H. Li, R. W. Erwin, J. Z .Lin, R. N. Shelton and P.Klavins, J. Appl. Phys., 67 (1990) 4533.

[4] J. Zaretsky, C. Stassis, A. I. Goldman, P. C. Canfield, P. Dervenagas, B. K. Cho and D. C. Johnston, Phys. Rev., B51 (1995) 678.

[5] S. K. Sinha, J. W. Lynn, T. E. Grigereit, Z. Hossain, L. C. Gupta, R. Nagarajan, C. Godard, Phys.Rev., B51 (1995) 681.

[6] A. Morrish, The physical principles of magnetism (J. Wiley, New York 1965).

[7] T. Krzysztoń, J. Magn. Magn. Materials, 15-18 (1980) 1572 and Phys. Lett., 104A (1984) 225.

[8] W. Thomlinson, G. Shirane, D. E. Moncton, M. Ishikawa, Ø. Fischer, J. Appl. Phys., 50 (1979) 1981 and Phys. Rev., B23 (1981) 4455

[9] T. Krzysztoń, Phys.Lett., A190 (1994) 196.

[10] M. Tinkham, Introduction to superconductivity, chapter 5, eqn (5-37), (McGraw-Hill Inc.,New York,1975).

[11] L. N. Bulaevskii, M. Ledvij and V. G. Kogan, Phys. Rev., B46 (1992) 366.

[12] J. R. Clem and M. W. Coffey, Phys. Rev., B42 (1990) 6209.

[13] M. Tachiki, S. Takahasi, Solid State Commun., 70 (1989) 291.

[14] S. Chakravarty, B. I. Ivlev, Y. N. Ovchinnikov, Phys. Rev., B42 (1990) 2143.

[15] E. H. Brandt, Physica, C195 (1992) 1.

[16] B. I. Ivlev, Y. N. Ovchinnikov, R. S. Thompson, Phys. Rev., B44 (1991) 7023.

[17] P. Tekiel, to be published in Z.Phys.B 
[18] G. Blatter, M. V. Feigelman, V. B. Geshkenbein, A. I. Larkin, Rev. Mod. Phys, 66 (1994)1125. 


\section{FIGURE CAPTIONS}

Fig.1. Schematic drawing of a piece of a layered superconductor. Hatched areas $(n, n+1)$ represent superconducting layers with thickness $\mathrm{d}_{s}$. Bold arrows represent magnetic moments of RE ions lying in the isolating layers of thickness $d_{i}$. The reference frame and the crystallographic axes are shown.

Fig.2. Single vortex line lying in the a-b plane and the induced spin-flop domain along its core.

Fig.3. Single vortex activation.

Fig.4. Activated bundle of vortices. 
This figure "fig1.jpg" is available in "jpg" format from: http://arxiv.org/ps/cond-mat/9711063v1 
This figure "fig2.jpg" is available in "jpg" format from: http://arxiv.org/ps/cond-mat/9711063v1 
This figure "fig3.jpg" is available in "jpg" format from: http://arxiv.org/ps/cond-mat/9711063v1 
This figure "fig4.jpg" is available in "jpg" format from: http://arxiv.org/ps/cond-mat/9711063v1 